\title{
TOTAL THYROIDECTOMY
}

\author{
Frequency of hypocalcemia after total thyroidectomy
}

Dr. Muhammad Sajid, Dr. Sarwat Bibi, Dr. Junaid Misbah.

ABSTRACT...... Objective: To find out frequency of hypocalcemia after total thyroidectomy. Study design: Descriptive study. Methods: 60 patients undergoing total thyroidectomy for multinodular goiter from January 2010 to December 2010 . Evaluation of results was done by analyzining the data in SPSS V-17. Results: In 60 patients undergoing total throidectomy, only one patient (1.67\%) developed permanent hypocalcemia and 11 patients (18.3\%) developed transient hypocalcemia. Conclusions: Total thyroidectomy can safely be performed in patients with multinodular goiter with very low risk of permanent hypocalcemia.

Key words: Hypocalcemia, Total Thyroidectomy

Article Citation

Sajid M, Bibi S, Misbah J. Total Thyroidectomy; Frequency of hypocalcemia after total thyroidectomy. Professional Med J 2013;20(3):433-438.

\section{INTRODUCTION}

Multinodular goiter is one of the commonest thyroid diseases encountered in the practice of surgery. Surgery is the main stay of treatment for most of the benign and all of the malignant conditions of thyroid. The extent of surgery varies for the benign as well as the malignant thyroid diseases. Various surgical options available are lobectomy, hemithyroidectomy, subtotal thyroidectomy, near total thyroidectomy and total thyroidectomy ${ }^{1}$.

Total thyroidectomy is currently the preferred treatment for thyroid cancer, multinodular goiter and graves disease and among patients with increased risk of recurrence.Total thyroidectomy is safe and is associated with a low incidence of disabilities. Total thyroidectomy seems to be the optimal procedure when surgery is indicated for graves disease and multinodular goiter as total thyroidectomy has the advantage of immediate and permanent cure and no recurrence ${ }^{2}$.

Despite the fact that total thyroidectomy is a more involved procedure that exposes more parathyroid glands and recurrent laryngeal nerve to surgical risks than unilateral thyroid lobectomy, it is an inherently safe procedure. Local complications such as recurrent laryngeal nerve paralysis and wound complications can be expected to occur in rates near $1 \%$, while post operative hypoparathyroidism may occur in approximately $6 \%$ of cases.Neither nodal dissection nor parathyroid reimplantation seems to affect the risk of postoperative hypocalcemia ${ }^{3}$.

Post operative hypocalcaemia is one of the complications of thyroidectomy ${ }^{4}$. The pathogenesis of hypocalcaemia after thyroidectomy is not completely understood. Hypocalcaemia after thyroidectomy has been most commonly attributed to parathyroid insufficiency related to injury, devascularisation, or inadvertent excision of the parathyroid glands. Other causative mechanisms that have been implicated in the pathophysiology of post-thyroidectomy hypocalcaemia include calcium uptake by bone in patients with thyrotoxic osteodystrophy, parathyroid suppression from increased calcium restored from the bone of patients with hyperthyroidism, transient preoperative haemodilution with increased renal excretion of calcium, increased release of calcitonin as a result of thyroid manipulation and autoimmunerelated fibrosis of the blood supply to parathyroid glands ${ }^{5}$.

It can be transitory or, in some cases, permanent. It is a 
very unpleasant situation for the patient. When intensive, it can be life threatening and is therefore a very serious complication in view of the preoperative diagnosis. Tetany and paraesthesia such as tingling around the mouth and in the distal extremities are commonly seen in hypocalcaemia. These symptoms were mainly reported in $15 \%$ of patients after sub-total thyroidectomy and $75 \%$ of patients after total thyroidectomy, with no case reported after lobectomy. Hypocalcemia after thyroidectomy is the most common postoperative complication $^{6}$.

Temporary hypoparathyroidism is defined as fall in corrected serum calcium levels below $8 \mathrm{gm} / \mathrm{dl}$, and/or the need for calcium supplementation.Permanent hypoparathyroidism is defined as the need for oral vitamin $D$ and/or calcium supplementation six months following surgery to maintain a normal serum calcium concentration. There is extremely low complication rate especially when using the technique of capsular dissection, staying close to the thyroid gland and preserving the blood supply to the parathyroid glands.By careful dissection and appropriate surgical technique total thyroidectomy can be performed as safely as near total thyroidectomy ${ }^{7}$.

We do total thyroidectomy for multinodular goiter in all patients. It is a safe procedure and complication rate is not significantly higher than other procedures used for multinodular goiter.

In this study we present our experience of 60 patients from January 2010 to December 2010. In all the patients, total thyroidectomy for multinodular goiter was done. Patients in whom near total or subtotal thyroidectomy was done were not included in this study.

\section{PATIENTS AND METHODS}

This study was conducted in surgical unit IV,DHQ hospital, Faisalabad from January 2010 to December 2010.Patients above 12 years of age in whom thyroidectomy was indicated due to multinodular goiter, carcinoma thyroid or recurrent goiter were included. Total of 60 patients were included who underwent total thyroidectomy for multinodular goiter by selected teams of surgeons in D.H.Q. hospital Faisalabad.

Preoperative serum calcium levels were obtained from hospital laboratory reported by pathologist to exclude hypocalcemia due to any other cause. During surgery all parathyroid glands were identified and saved. If any gland was dissected accidently, it was autotransplanted in sternocleidomastoid muscle. Individual branches of inferior thyroid artery were ligated instead of main trunk of artery to prevent devascularisation of glands. Postoperatively serum calcium levels were obtained 24hrs, 48hrs, 3months and then 6months after surgery. Patients were also examined clinically for signs and symptoms of hypocalcemia. Hypocalcaemic patients were kept admitted and treated with IV calcium and monitoring of serum calcium was done till discharge.

If hypocalcemia was reversed within 3 months it was labeled as transient hypocalcemia and if serum calcium levels did not revert even after 6 months it was labeled as permanent hypocalcemia.

The record of hypocalcemia following total thyroidectomy was noted on data sheets and analyzed by using SPSS-V 17.

\section{RESULTS}

Out of 60 cases operated $56(93.3 \%)$ were female and $4(6.67 \%)$ were male. Minimum age was 17 years and maximum age was 55 years. Out of 60 patients, $53(88.3 \%)$ were of benign multinodular goiter, $5(8.33 \%)$ were of carcinoma of thyroid and $2(3.33 \%)$ were of recurrent goiter. 


\begin{tabular}{|l|c|}
\hline $\begin{array}{l}\text { Gender } \\
\text { Male }\end{array}$ & $4(6.67 \%)$ \\
Female & $56(93.3 \%)$ \\
\hline Age & $17-55$ years \\
\hline Indication of surgery & \\
Multinodular goiter & $53(88.3 \%)$ \\
Carcinoma thyroid & $05(8.33 \%)$ \\
Recurrent goiter & $02(3.33 \%)$ \\
\hline
\end{tabular}

Table-I.

Out of 60 patients, 1 patient (1.67\%) developed permanent hypocalcemia and 11 patients (18.3\%) developed transient hypocalcemia. Patients with transient hypocalcemia recovered and became normocalcemic in 2 to 3 months. In one patient hypocalcemia persisted upto 9 months and was labeled as permanent hypocalcemia.

\begin{tabular}{|c|c|c|c|c|}
\hline \multirow{2}{*}{} & \multicolumn{3}{|c|}{ Hypocalcemia } & \multirow{2}{*}{ Total } \\
\cline { 2 - 4 } & Transient & Permanent & None & \\
\hline Male & $1(1.67 \%)$ & $0(0 \%)$ & $3(5.0 \%)$ & $4(6.67 \%)$ \\
\hline Female & $10(16.7 \%)$ & $1(1.67 \%)$ & $45(75.0 \%)$ & $56(93.3 \%)$ \\
\hline Total & $11(18.3 \%)$ & $1(1.67 \%)$ & $48(80 \%)$ & $60(100 \%)$ \\
\hline \multicolumn{3}{|c|}{ Table-Il. } \\
\end{tabular}

Histopathological report of all patients did not reveal any parathyroid tissue in the specimen. All hypocalcaemic patients were symptomatic and presented with perioral numbness, Chovostick's and Trousseau's signs. Cardiac arrhythmias, stridor and other signs were absent.

\section{DISCUSSION}

Surgical resection is the treatment of choice for majority of patients with benign multinodular goiter and all malignant tumors of thyroid. Current indications for surgery are compression induced symptoms, suspected malignancy, hyperthyroidism and cosmesis.Surgical options for the management of multinodular goiter include bilateral subtotal thyroidectomy, near total thyroidectomy and total thyroidectomy ${ }^{8}$.

Total thyroidectomy is accepted worldwide as a standard surgical procedure for the management of benign multinodular goiter disease. Hypocalcemia is the most common complication of this procedure. The reported incidence of hypocalcemia is around $20-30 \%$ with symptoms ranging from mild paraesthesia, tingling to more severe cramps, tetany, convulsions. Nide for hypocalcemia typically occurs at around 2448 hrs post operatively $^{9}$.

In several series, the incidence of hypocalcemia varied from $10.6 \%$ to $50 \%$ but may be as high as $83 \%{ }^{10}$. In other study, 33.3\% patients developed hypocalcemia after thyroidectomy. 1.2\% developed permanent hypocalcemia in total thyroidectomy ${ }^{11}$. In one study permanent hypocalcemia was found in 16.77\%, transient hypocalcemia was in $39 \%$ patients $^{12}$. In another local study,permanent hypocalcemia was noted in $39 \%{ }^{13}$.

In patients experiencing significant hypocalcemia, the most probable cause is hypoparathyroidism secondary to trauma, devascularisation or inadvarent excision of one or more parathyroid glands during surgery. In most patients hypocalcemia after thyroid surgery is self limiting but in some it may be potentially dangerous ${ }^{14}$.

In our study patients were classified hypocalcemic if serum calcium level was below $8 \mathrm{mg} / \mathrm{dl}$. Serum calcium levels were measured preoperatively, $24 \mathrm{hrs}$ after surgery, $48 \mathrm{hrs}$ after surgery, three \& six months after surgery. If serum calcium levels returned to normal in three months, it was classified as temporary hypocalcemia \& if serum calcium levels didn't return to normal after six months, it was labeled as permanent hypocalcemia.

In study done by sokouti $M$ et al the conclusion was that transient hypocalcaemia was seen in 18 patients 
(27.7\%) whereas permanent hypocalcaemia was not seen in any of their patients who underwent total thyroidectomy for carcinoma thyroid and mean time for transient hypocalcaemia correction was two week ${ }^{15}$. In one study, hypocalcemia was observed in $41.2 \%$ of all cases persisting in $5.6 \%$ at last follow up thus patient probably developed permanent hypocalcemia $^{16}$. In another study, all patients were euthyroid at time of surgery. Hypocalcemia was observed in 16 patients. In all 16 hypocalcemic patients, serum parathyroid hormone level was low but usually became normal in 2-3 months. All hypocalcemic patients recovered and became normocalcemic between 2-3 months. In one patient, hypocalcemia persisted upto 10 months, it was noticed that $25 \%$ patients of thyroid carcinoma developed hypocalcemia ${ }^{17}$. In our study, permanent hypocalcemia developed in 1 patient out of 60 thus making a percentage of $1.67 \%$ and transient hypocalcemia developed in 11 patients making a percentage of $18.3 \%$. Thus the cause appears to be trauma, devascularisation or inadvertent excision of parathyroid glands.

This injury can be prevented if thyroidectomy is done by intracapsular approach. It has also been proved that if branches of inferior thyroid artery are individually ligated inside the capsule of thyroid gland instead of ligating the main trunk in continuity, then more patients can be prevented from developing hypocalcemia. Upper parathyroid glands are usually constant in position and lower two parathyroid glands are variable in location \& in $60 \%$ of cases, they are located away from thyroid gland. So in total thyroidectomy, they usually escape injury ${ }^{18}$. Our study outcome was better when compared to Rosato $\mathrm{L}$ where the hypocalcaemia was reported to be transient in $14 \%$ and definitive in $2.2 \%$. This less incidence of hypocalcaemia in our study was probably because of the fact that we stuck to the policy of identification of parathyroids in all of the cases, not tying the main trunk of inferior thyroid artery and only tying the branches close to the surface of thyroid thus sparing the blood supply to the parathyroids with less likelihood of parathyroid shock. Whether tying or not tying the inferior thyroid artery makes any difference for the parathyroid functioning is debatable in the literature however we found it to be the main reason for a low incidence of hypocalcemia in our study.

In our study, surgical dissection of thyroid gland took place after identification \& preservation of both laryngeal nerves and superior, inferior parathyroid glands.In all patients, we identified 3-4 parathyroid glands, left them in situ with their own blood supply. If we inadvertently removed or devascularised a parathyroid gland, we auto transplanted the gland into ipsilateral sternocleidomastoid muscle. We drained the neck with suction drains for 48hrs and patients were usually discharged 2 days after surgery.

A very interesting observation was the decrease of serum calcium level levels in the immediate postoperative periods, showing a statistical significance at the second post operative day when compared to preoperative calcium levels.

Another very significant observation is the low reliability of clinical observations when evaluating postoperative hypocalcaemia in thyroidectomies. On the other hand, when symptomatology occurred in the early postoperative period, we observed low or very close to low normal calcium levels. In the late postoperative period, we saw a significant increase of symptoms of patients with normal calcium level. This curious finding can be easily explained by the nonspecific nature of these symptoms and the possibility that they were suggested to the patients by insistent interrogation, as has also been postulated by other authors. All symptomatic patients presented with peri oral numbness, tingling, Chovostick's sign and Trousseau's sign.Cardiac arrhythmias, stridor and other signs were absent. At this time, we think that it is very important to confirm the clinical suggestion of 
hypocalcaemia with determination of calcium levels to avoid unnecessary treatment.

We have found that it is a very safe procedure with a good and safe technique.

It has the major advantages that chances of recurrence are very low; morbidity due to recurrent thyroid surgery is avoided and underlying malignancy if any is treated in the same sitting.

\section{CONCLUSIONS}

Total thyroidectomy is good and safe therapeutic option for patients of thyroid diseases especially multinodular goiter. The complication related to parathyroid gland although rare but is still a considerable issue. Evaluation of hypocalcaemia with clinical suggestion is not reliable tool and must be confirmed with serum calcium levels. There is gradual decrease in serum calcium level in early postoperative period, which is temporary and becomes stable after three months. If individual branches of inferior thyroid artery are ligated instead of main trunk, blood supply of parathyroid glands can be saved.

Total thyroidectomy removes the disease process completely, lowers local recurrence rates and avoids the substantial risks of reoperative surgery. Total thyroidectomy is safe and can be carried out with low complication rates that are equal to world's centers of excellence.

\section{Copyright(c) 18 Jan, 2013.}

\section{REFERENCES}

1. Sheikh IA, Waleem SS, Haider IZ, Haroon A, Ashfaq M. Total thyroidectomy as primary elective procedure in multinodular thyroid disease. J Ayub Med Coll Abbottabad. 2009;21(4):57-9.

2. Efremidox El, Papageorgious MS, Liratzopoulos N, Manolas KJ. The efficacy and safety of total thyroidectomy in the management of benign thyroid disease: a review of 932 cases. Can J Surg. 2009; 52:39-44.
3. Bhattacharyya N, Fried MP. Assessment of morbidity and complications of total thyroidectomy. Arch Otolaryngeal Head Neck Surg 2002;128:389-92.

4. Surgery of the neck.In: Farquharson M, Moran B, Farquharson's textbook of operative general surgery. 9th ed. New York: Hodder Arnold; 2005.p.155-78.

5. Sturniolo G, Schiavo MG, Tonante A, D'Alia C, Bonano L. Hypocalcemia and hypoparathyroidism after total thyroidectomy: a clinical biological study and surgical consideration. Int J Surg Investig 2000;2:99105.

6. Proczko-Markuszewska M, Kobiela J, Stefaniak T, Larkinski AJ, Sledzinski Z. Postoperative PTH measurement as a predictor of hypocalcemia after thyroidectomy. Acta Chir Belg. 2010;110:40-4.

7. Ozbas S, Kocak S, Aydintug S, Cakmak A, Demirkiran MA, Wishart GC. Comparison of the complications of subtotal, near total and total thyroidectomy in the surgical management of multinodular goiter. Endocrine J 2005;52:199-205.

8. Sciumi C, Geraci G, Pisello F, Facella T, Li voles F, Licata $A$, et al. Complications in thyroid surgery; sympyomatic postoperative hypoparathyroidism incidence, surgical technique and treatment. Aun Ital Chic. 2006;77:115-22.

9. Simon $G$, Janathan $S$. Evidence for the role of perioperative PTH measurement after total thyroidectomy as a predictor of hypocalcemia. World J Surg 2008;32:1367-73.

10. Abboud B, Sargi Z, Akham M, Sleilatry F. Risk factors for post thyroidectomy hypocalcemia. J Am Coll Surg. 2002;195:456-67.

11. Di Fabio F, Casella C, Bugari G, lacobello C, Salemi B. Identification of patients at low risk for thyroidectomy related hypocalcemia by intraoperative quick PTH. World J Surg.2006;30:1428-33.

12. Qari F. Estimation of ionized calcium levels after thyroidectomy at King Abdul Aziz University Hospital. Kuwait Med J. 2005;37:169-72.

13. Rajput A, Samad A, Channa GA, Khanjada TW, Ujjan I. 
Hypocalcemia; a genuine threat after thyroidectomy. PakJ Surg.2009;25:6-9.

14. Chia SH, Weisman RA, Piex D, Kelly C, Dilmann WH, Ochoff $L A$. Prospective study of perioperative factors predicting hypocalcemia after thyroid and parathyroid surgery. Arch Otolaryngeal Head Neck Surg.2006; 132:41-5.

15. Sokouti M, Montazeri V, Golzari S. The incidence of transient and permanent hypocalcaemia after total thyroidectomy for thyroid cancer. Int $\mathrm{J}$ Endocrinol Metab 2010; 1: 7-12.

16. Warren FM, Andersen PE, Wax MK, Cohen Jl.
Intraopertive parathyroid hormone levels in thyroid and parathyroid surgery. Laryngoscope 2002; 112:1866-70.

17. Iqbal M, Subhan A, Baig MS, Shah MS. Frequency of hypocalcemia in total thyroidectomy. J Surg Pak(Int) 2010;15:87-91.

18. Ozbas S, Kocak S, Aydintug S, Cakmak A, Demirkiran MA, Wishart GC. Comparison of the complications of subtotal, near total and total thyroidectomy in the surgical management of multinodular goiter. Endocr J. 2005;52:199- 205.

\section{AUTHOR(S):}

1. DR. MUHAMMAD SAJID MBBS, FCPS (Surgery)

Associate Professor of Surgery

Punjab Medical College, Faisalabad.

2. DR. SARWAT BIBI

Registrar Surgery

DHQ Hospital (PMC), Faisalabad.

3. DR. JUNAID MISBAH

Registrar Surgery

DHQ Hospital (PMC), Faisalabad.
Correspondence Address:

Dr. Muhammad Sajid

MBBS, FCPS (Surgery)

Associate Professor of Surgery

Punjab Medical College, Faisalabad.

sajid_sh@msn.com

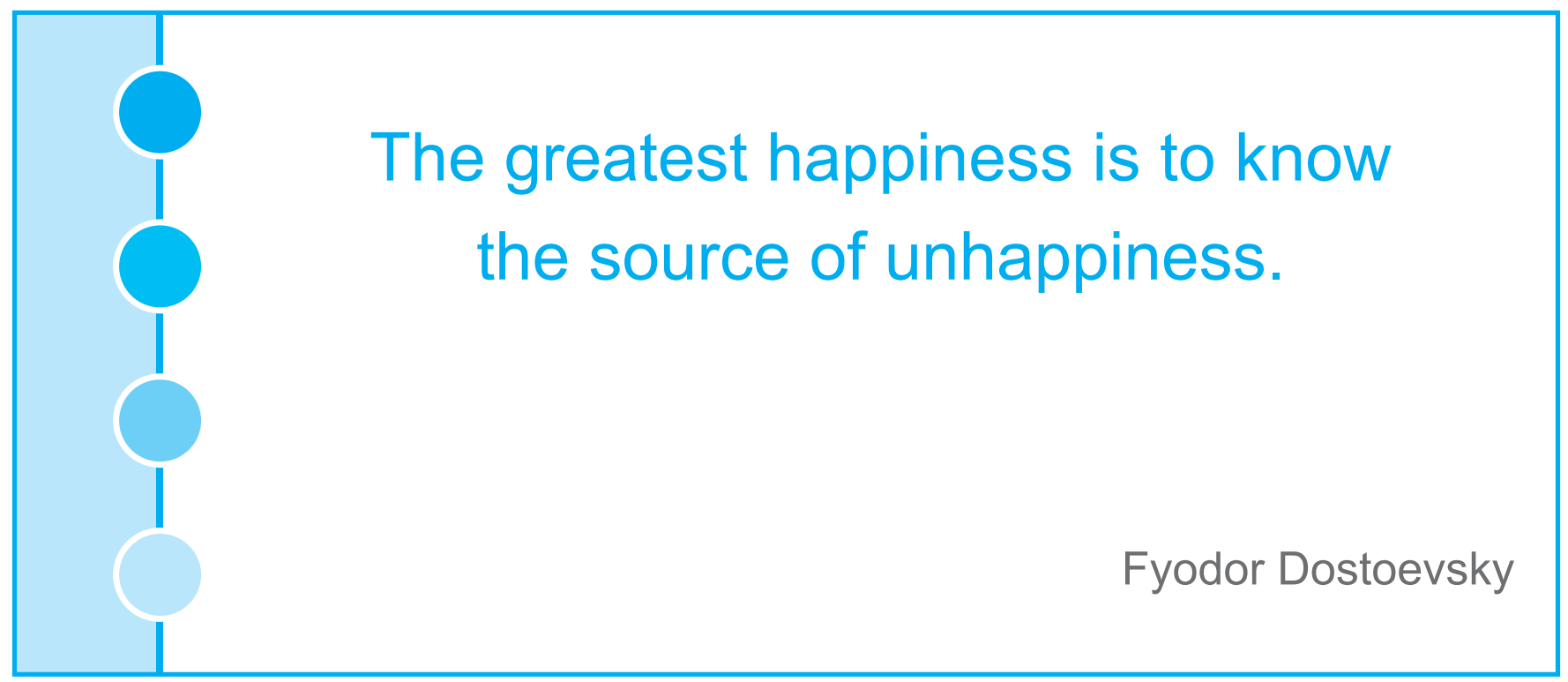

